antagonist PP2A-B55 $\delta$ via the Gwl-Ensa-Arpp19 pathway (21-23). Perhaps Cdk1 itself could inactivate PP2A-B'56 once it reaches maximal activity in metaphase. By identifying XErp1 as a mitotic $\mathrm{APC} / \mathrm{C}$ inhibitor, our studies provide a framework for understanding how different regulatory modules composed of phosphorylations of Cdc20, APC/C, XErp1, and possibly PP2AB'56 are interconnected to create oscillatory Cdk1 activity driving cell cycle progression.

References and Notes

1. T. Duncan, T. T. Su, Curr. Biol. 14, R305 (2004).

2. R. S. Hartley, R. E. Rempel, J. L. Maller, Dev. Biol. 173, 408 (1996).

3. J. L. Maller et al., Cell cycle transitions in early Xenopus development. Novartis Found. Symp. 237, 58 (2001)

4. ]. E. Ferrell ]r., M. Wu, ]. C. Gerhart, G. S. Martin, Mol. Cell. Biol. 11, 1965 (1991).

5. P. Clute, Y. Masui, Dev. Growth Differ. 34, 27 (1992).
6. K. Ohsumi, A. Koyanagi, T. M. Yamamoto, T. Gotoh, T. Kishimoto, Proc. Natl. Acad. Sci. U.S.A. 101, 12531 (2004).

7. A. Schmidt et al., Genes Dev. 19, 502 (2005).

8. D. Inoue, M. Ohe, Y. Kanemori, T. Nobui, N. Sagata, Nature 446, 1100 (2007).

9. ]. Liu, B. Grimison, A. L. Lewellyn, J. L. Maller J. Biol. Chem. 281, 34736 (2006).

10. J. Liu, J. L. Maller, Curr. Biol. 15, 1458 (2005).

11. T. Nishiyama, K. Ohsumi, T. Kishimoto, Nature 446, 1096 (2007).

12. N. R. Rauh, A. Schmidt, ]. Bormann, E. A. Nigg, T. U. Mayer, Nature 437, 1048 (2005).

13. See supplementary materials on Science Online.

14. J. Q. Wu et al., Proc. Natl. Acad. Sci. U.S.A. 104, 16564 (2007)

15. M. Isoda et al., Dev. Cell 21, 506 (2011)

16. Q. Wu et al., Curr. Biol. 17, 213 (2007).

17. N. Sagata, N. Watanabe, G. F. Vande Woude, Y. Ikawa, Nature 342, 512 (1989)

18. C. M. Smith, E. Radzio-Andzelm, P. Madhusudan, P. Akamine, S. S. Taylor, Prog. Biophys. Mol. Biol. 71, 313 (1999).

19. C. D. Ashby, D. A. Walsh, J. Biol. Chem. 248, 1255 (1973).

20. T. Chijiwa et al., J. Biol. Chem. 265, 5267 (1990).
21. A. Gharbi-Ayachi et al., Science 330, 1673 (2010).

22. S. Mochida, S. L. Maslen, M. Skehel, T. Hunt, Science 330, 1670 (2010).

23. ]. Yu, Y. Zhao, Z. Li, S. Galas, M. L. Goldberg, Mol. Cell 22, 83 (2006).

Acknowledgments: We thank D. Bertinetti, A. Bürkle, J. Gannon, T. Hunt, F. W. Herberg, T. Lorca, S. Mochida, and N. Sagata for reagents; the team of D. Schopper and G. Mende for excellent animal care; and the Mayer group and B. Novak for discussions. The authors declare no competing financial interests. Supported by the Doc-fForte program of the Austrian Academy of Science and by the Collaborative Research Center 969 of the German Research Foundation (DFG).

\section{Supplementary Materials}

www.sciencemag.org/cgi/content/full/science.1228394/DC1

Materials and Methods

Figs. S1 to S5

Movie $\mathrm{S1}$

References (24-27)

3 August 2012; accepted 4 September 2012

Published online 27 September 2012;

$10.1126 /$ science.1228394

\title{
Maturation-Dependent HIV-1 Surface Protein Redistribution Revealed by Fluorescence Nanoscopy
}

\section{Jakub Chojnacki, ${ }^{1}$ Thorsten Staudt, ${ }^{2}$ Bärbel Glass, ${ }^{1}$ Pit Bingen, ${ }^{2}$ johann Engelhardt, ${ }^{2}$ Maria Anders, ${ }^{1}$ jale Schneider, ${ }^{2}$ Barbara Müller, ${ }^{1}$ Stefan W. Hell, ${ }^{2,3}$ Hans-Georg Kräusslich ${ }^{1 *}$}

Human immunodeficiency virus type 1 (HIV-1) buds from the cell as an immature particle requiring subsequent proteolysis of the main structural polyprotein Gag for morphological maturation and infectivity. Visualization of the viral envelope (Env) glycoprotein distribution on the surface of individual HIV-1 particles with stimulated emission depletion (STED) superresolution fluorescence microscopy revealed maturation-induced clustering of Env proteins that depended on the Gag-interacting Env tail. Correlation of Env surface clustering with the viral entry efficiency revealed coupling between the viral interior and exterior: Rearrangements of the inner protein lattice facilitated the alteration of the virus surface in preparation for productive entry. We propose that Gag proteolysis-dependent clustering of the sparse Env trimers on the viral surface may be an essential aspect of HIV-1 maturation.

$\mathrm{T}$ The lipid envelope of human immunodeficiency virus type 1 (HIV-1) carries trimers of dimers of the viral envelope (Env) surface glycoprotein gp120 and the transmembrane protein gp41 (Fig. 1). Env proteins mediate virus binding and fusion with the target cell membrane and are essential for infectivity. Env incorporation into the virus particle is thought to occur through interaction of the gp41 C-terminal tail with the viral structural polyprotein Gag (1-3).

\footnotetext{
${ }^{1}$ Department of Infectious Diseases, Virology, Heidelberg University, Im Neuenheimer Feld 324, 69120 Heidelberg, Germany. ${ }^{2}$ Optical Nanoscopy Division, German Cancer Research Center (DKFZ), Im Neuenheimer Feld 280, 69120 Heidelberg, Germany, and BIOQUANT, Im Neuenheimer Feld 267, 69120 Heidelberg, Germany. ${ }^{3}$ Department of NanoBiophotonics, Max Planck Institute for Biophysical Chemistry, Am Fassberg 11 , 37077 Göttingen, Germany.
}

*To whom correspondence should be addressed. E-mail: hans-georg.kraeusslich@med.uni-heidelberg.de
Biochemical and cryo-electron microscopy (cryoEM) studies revealed the presence of only 7 to 14 Env trimers per virus particle $(4,5)$, whereas related lentiviruses and other enveloped viruses generally have a much higher surface density of viral entry proteins $(6-8)$. Concomitant with budding, the immature hexameric Gag lattice underneath the viral membrane is dissolved into its constituent domains via HIV-1 protease (PR)mediated cleavage. This results in formation of the mature infectious HIV-1 structure with its characteristic cone-shaped capsid (Fig. 1). Proteolysis and morphological maturation activate viral replication enzymes and render the metastable capsid shell ready for uncoating, thereby converting the virus from an assembly to an infection mode (9).

Immature or partially matured HIV-1 particles display reduced entry efficiency without apparent differences in Env protein structure or composition $(10,11)$. This effect could be caused by the stiffness of the immature Gag lattice underneath the viral membrane restricting membrane fusion (12) or by alterations in Env lateral mobility that may prevent clustering of sparsely distributed Env trimers. Superresolution fluorescence microscopy provides an opportunity to analyze subviral structures on a statistically significant number of particles. Here, we used stimulated emission depletion (STED) microscopy (13) to investigate the distribution of Env molecules on the surface of individual HIV-1 particles.

Env was immunolabeled with the human monoclonal antibodies 2G12 (14) or b12 (15), which recognize the gp120 domain. Antibodyinduced clustering was avoided by using purified Fab fragments (fig. S1). To identify the position of individual HIV-1 particles in the image, we used the viral accessory protein $\mathrm{Vpr}$ tagged with enhanced green fluorescent protein (eGFP) (16) as a "counterstain" reference. Visualization of HIV-1 in the confocal mode of our STED microscopy setup showed blurred spots in the eGFP (green) channel (Fig. 1A). An overlay with a confocal channel mapping the Atto 565-labeled 2G12 Fab immunocomplexes (red) displayed colocalization for $\sim 90 \%$ of the eGFP signals, without disclosing any subviral details (Fig. 1A). Visualization of the same area with the Atto 565 signal recorded in the STED mode, resulting in about five times higher resolution (40 nm; fig. S2), revealed distinct, small, punctuate signals colocalizing with eGFP-labeled particles (Fig. 1B). A similar staining pattern was observed when Env was detected with b12 Fab (fig. S3). Fab-derived signals were not detected upon immunostaining of particles lacking Env (Fig. 1C and fig. S3B), demonstrating the specificity of staining. STED analysis revealed differences between the Env distributions on mature and immature particles. Whereas most mature particles displayed a single Env signal or focus (Fig. 1B), the majority of immature particles were found to exhibit two or more Env foci (Fig. 1D 
and fig. S3). The distance between Env foci on individual particles did not exceed $150 \mathrm{~nm}$, consistent with the reported average particle diameter of HIV-1 (17).

To ensure that the detection of multiple Env foci did not result from particle aggregation unrecognizable in the diffraction-limited confocal mode, we visualized the eGFP.Vpr signal of adhered particles using STED microscopy (fig. S4): $97.2 \%$ of mature and $97.6 \%$ of immature particles represented individual particles, consistent with previous observations based on diffusion velocity measurements (18). We used STED microscopy to determine whether the higher number of Env foci on immature particles indicated differences in Env distribution or simply a higher number of Env molecules by comparing the signal of virus-associated Env molecules with the signal from defined references. Reference samples included Atto 565-labeled secondary Fab fragments (Fig. 2A, black bars), as well as monomeric (light gray bars) or trimeric (dark gray bars) recombinant HIV-1 Env proteins bound to 2G12 Fab and Atto 565-labeled secondary Fab. Side-by-side comparison of Env fluorescence signals on HIV-1 particles with those of reference samples yielded an average of seven and eight Env trimers on mature and immature HIV-1, respectively (Fig. 2B), consistent with published reports $(4,5)$. These results were supported by quantitative immunoblotting of bulk preparations of mature and immature particles, which showed no significant difference in their respective Env content (Fig. 2D and fig. S5). Finally, we analyzed the relative amount of Fab fragments bound to HIV-1 in solution by immunoblot analysis of particle-Fab complexes. Mature and immature particles featured comparable amounts of bound Fab (Fig. 2E). Thus, the observed differences in STED signal distribution were not caused by differences in Env content or Fab accessibility.

To quantify the observed difference between mature and immature HIV-1 particles, we manually classified the Env signals associated with individual eGFP-labeled particles into three distribution patterns: (i) single focus, (ii) two foci, and (iii) more than two foci (Fig. 3A and fig. S6). These patterns were indistinguishable by confocal microscopy (Fig. 3A, right column). Whereas single Env foci were found in $\sim 70 \%$ of the mature particles, they were observed in less than $30 \%$ of their immature counterparts (Fig. 3B and table S1). The same difference was seen when Env was expressed from a different plasmid than the remaining HIV-1 proteins. This so-called pseudotyping led to an increase in Env incorporation, but yielded a similar Env surface distribution with $\sim 70 \%$ of mature particles and less than $30 \%$ of immature particles exhibiting a single focus (Fig. 3C and table S1). Env signal intensities for virus particles displaying different numbers of foci were not significantly different, confirming that multi-

A

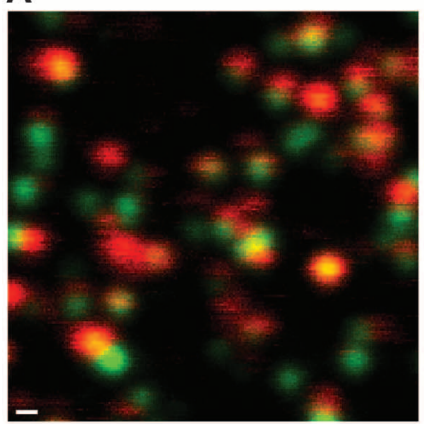

C

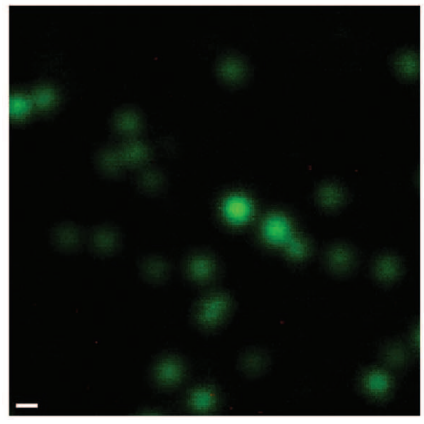

B
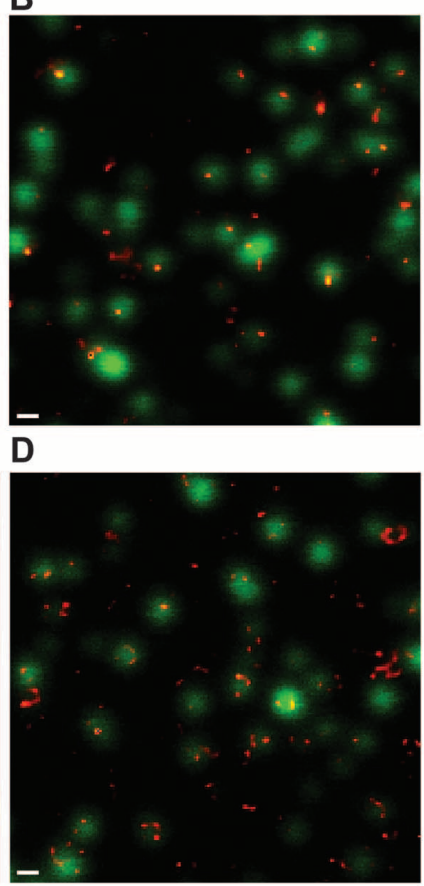

E
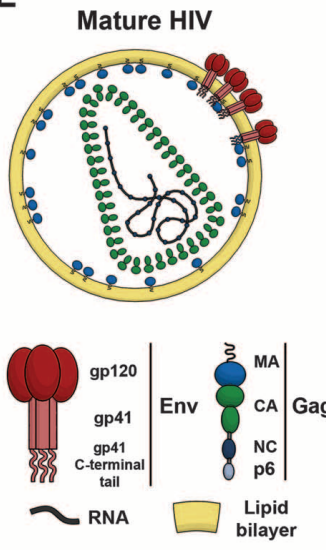

Immature HIV

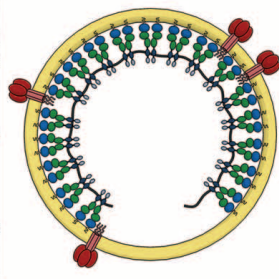

Fig. 1. HIV-1 Env distribution visualized by STED microscopy. Purified HIV-1 particles, containing eGFP. Vpr (green), were stained for Env (orange). Images were acquired using the STED setup with the eGFP. Vpr signal recorded in the standard confocal mode to define the localization of particles. Scale bars: $200 \mathrm{~nm}$. (A) Env signal of mature particles acquired in the confocal microscope mode. (B) The same field of view as in (A), with the Env signal acquired in STED mode. (C) Control Env(-) particles with the Env signal acquired in STED mode. (D) Env signal of immature particles acquired in STED mode. (E) Schematic illustration of the mature and immature HIV particle structures. focal Env distribution patterns did not arise from an increased number of Env molecules on the particle surface (Fig. 2C).

Next, we extended the Env distribution analysis to HIV-1 variants displaying partial maturation defects due to mutation of specific Gag cleavage sites (fig. S7A). This included a variant with a processing defect between the matrix (MA) and capsid (CA) domains of Gag (MA-CA) and one in which all Gag cleavage sites except for the one between MA and CA were blocked (CA-p6). The covalent linkage of MA with the assembled CA domain in the MA-CA variant has been shown to retain the hexameric immature lattice and should keep the MA layer in the same position as in the immature particle. Conversely, the processed MA is released from the underlying lattice in the CA-p6 variant and should be free to move laterally (19). The expected Gag processing patterns were confirmed by immunoblotting of purified particles (fig. S7C). Analysis of Env distribution on the viral surface showed that the MA-CA variant resembled the immature phenotype, with $\sim 35 \%$ of particles exhibiting a single Env focus, whereas the CA-p6 variant displayed an intermediate phenotype more similar to mature HIV-1 (Fig. 3B and table S1). Thus, Env surface distribution of HIV-1 correlates with the processing of the MA domain of Gag, which lines the inner face of the viral membrane.

The dependence of HIV-1 Env surface distribution on MA processing suggested a role for the unusually long (151 amino acids) Env C-terminal tail (CT) that interacts with the underlying MA layer $(1-3,20)$. Deletion of Env $\mathrm{CT}$ and the consequent loss of Env interaction with the underlying Gag lattice may render Env distribution independent of the HIV-1 maturation status. We tested this hypothesis by comparing Env distribution patterns on mature and immature HIV-1 pseudotyped either with full-length Env or an Env CT-deleted variant $[\operatorname{Env}(\Delta \mathrm{CT})$; fig. S7B]. $\operatorname{Env}(\Delta C T)$ exhibited an immature-like Env distribution pattern independent of the maturation status of the virus. A single Env cluster was observed in less than $30 \%$ of particles for both immature and mature $\operatorname{Env}(\Delta \mathrm{CT})$ particles, and these numbers were indistinguishable from those obtained for immature HIV-1 with wildtype (wt) Env (Fig. 3C, fig. S8, and table S1). These results suggest that the Env CT is actively involved in Env clustering. Moreover, the observed maturation-dependent formation of a single Env cluster may require both disassembly of the underlying rigid Gag lattice and CTdependent Env interactions. This conclusion is supported by a previous report showing that the HIV-1 Env CT has the potential for self-association (21), which may be the driving force for the observed clustering.

To determine whether clustering of the sparse Env trimers on the HIV-1 surface is relevant for virus entry, we analyzed the infectivity and entry competence of mature HIV-1 particles, carrying either wild-type or C-terminally truncated Env. In 
agreement with published reports $(3,22)$, deletion of the C-terminal tail led to a highly significant drop in HIV-1 infectivity. This was observed in a single-round assay (Fig. 4A) and in an end-point titration assay (integrating multiple rounds of infection; Fig. 4B) in lymphocytic cell lines. To extend this analysis to immature particles, we performed entry assays, in which cytosolic delivery of HIV-1 contents after fusion is measured by activity of a virus-incorporated reporter protein (23). These experiments were performed with primary human lymphocytes, which are natural target cells of HIV-1. Consistent with the reduced infectivity (Fig. 4, A and B), truncation of the Env C-terminal tail also led to a significant decrease of fusion competence of mature HIV-1 (Fig. 4C). The entry competence was even lower for immature HIV-1 carrying wild-type Env, as observed previously (10), but truncation of the C-terminal tail partially rescued entry in this case (Fig. 4C). Thus, entry efficiency generally correlated with the observed Env clustering with the exception of immature HIV-1 with $\operatorname{Env}(\Delta \mathrm{CT})$. Env CT de- letion partially restored entry competence in this case, but did not affect Env clustering on free virus particles.

This phenotype could be explained by a delayed Env clustering upon engagement of the cellular receptor, which would be blocked by the immature Gag lattice in the presence of Env CT. We tested this hypothesis directly by dual-color STED microscopy of HIV-1 particles attached to $T$ cells, recording both the viral glycoprotein and the cellular receptor CD4 with nanoscale resolution. The cell-attached viral particles identified by their GFP signal were almost always associated with CD4 patches on the cell surface. Analysis of more than 60 individual HIV-1 particles per variant revealed a clear polarization of Env foci toward the CD4 patch, suggesting direct receptor engagement (Fig. 4, D to I, and fig. S9). The Env distribution patterns of cell-bound mature and immature particles carrying $\operatorname{Env}(\mathrm{wt})$ closely resembled the distributions observed for the respective cell-free viruses (Fig. 4, D, E, and I, and fig. S10). By contrast, virus particles car- rying $\operatorname{Env}(\Delta \mathrm{CT})$ displayed a shift from the predominantly multifocal distribution observed in free particles to a high degree of single foci for CD4-engaged particles (Fig. 4, F, G, and I, and fig. S10). This difference was observed for mature and immature particles carrying $\operatorname{Env}(\Delta \mathrm{CT})$, indicating that Env clustering can be induced by contact with the receptor, provided that the Env CT is deleted.

On the basis of these results, we propose that Env clustering into a single focus is important for efficient HIV-1 entry. The entry competence of HIV-1 directly correlated with its capacity to form Env clusters, and truncation of the Env C-terminal tail rescued both clustering and cytosolic entry of immature viruses in primary lymphocytes. An involvement of Env clusters in the fusion event has been previously proposed on the basis of structures observed at contact sites between particles and $\mathrm{T}$ cells by electron tomography (24). Env clustering may be particularly important for HIV-1 because of the low number of Env trimers in the viral membrane.
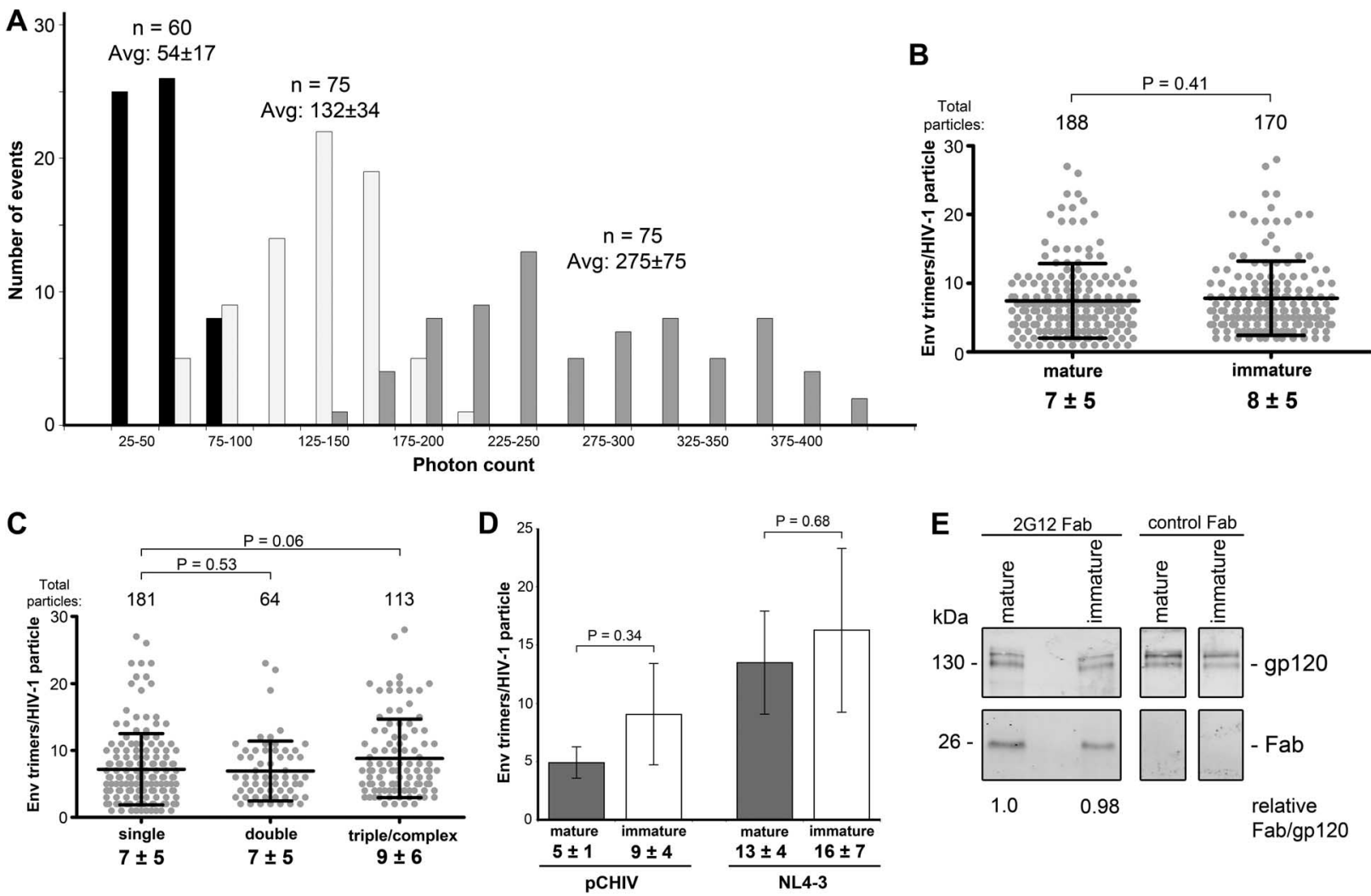

Fig. 2. Quantitation of Env trimers on mature and immature HIV-1. (A) Fluorescence intensity distribution of reference samples acquired by STED microscopy. Fab fragments were labeled with Atto 565. Black bars: anti-human Fab; light gray bars: recombinant monomeric Env stained with primary and secondary Fab; dark gray bars: recombinant trimeric Env stained with primary and secondary Fab. (B) The number of Env trimers on individual mature and immature HIV-1 particles was estimated from the average fluorescence intensity of the recombinant trimeric Env reference sample analyzed in parallel. Mean \pm SD values are shown with Wilcoxon rank-sum test for significance. (C) Env trimer incorporation classified according to Env distribution class and shown for the combined data of mature and immature HIV-1.

Mean \pm SD values are shown with Wilcoxon rank-sum test for significance. (D) Amounts of gp120 and CA or Gag in purified virus preparations from cells transfected with pCHIV or pNL4-3, respectively, were determined by quantitative immunoblotting. The number of Env trimers per particle was calculated by assuming an average of 2400 CA or Gag molecules per particle (26). Mean \pm SD values are shown with Wilcoxon rank-sum test for significance ( $n=4$ experiments). (E) A representative immunoblot showing Fab binding to mature and immature HIV-1, respectively. Purified particles were incubated with $2 \mathrm{G} 12$ or control Fab fragments and analyzed for Fab binding by quantitative immunoblotting. The ratio of gp120 to Fab in mature particles was set to 1 . 
Fig. 3. HIV-1 Env distribution patterns depending on Gag maturation state and the presence of Env CT. (A) Representative images of eGFP.Vpr (green) containing HIV-1 particles stained for Env (orange), displaying different Env distribution classes: (i) single Env focus, (ii) two Env foci, (iii, iv) three or more Env foci. Scale bars: $100 \mathrm{~nm}$. (B and C) Env distribution in different HIV-1 variants. Particles identified by eGFP.Vpr staining were classified into three Env distribution classes as exemplified in (A): single Env focus (black bars), two Env foci (light gray bars), three or more Env foci (dark gray bars). Data represent the mean of three independent blind counts by three different investigators with significance assessed by $\chi^{2}$ test for independence against a wild-type Env distribution at two degrees of freedom. (B) Mature (wt) particles compared to immature (PR-) particles or variants with mutations at distinct PR cleavage sites as described in the text. All derivatives carry Env(wt). (C) Comparison of mature and immature (PR-) particles pseudotyped with $\operatorname{Env(wt)~or~} \operatorname{Env}(\Delta \mathrm{CT})$, respectively.
A

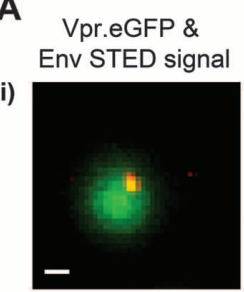

ii)

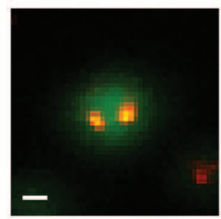

iii)

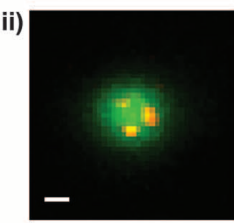

iv)

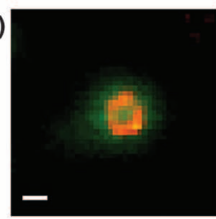

Env STED signal
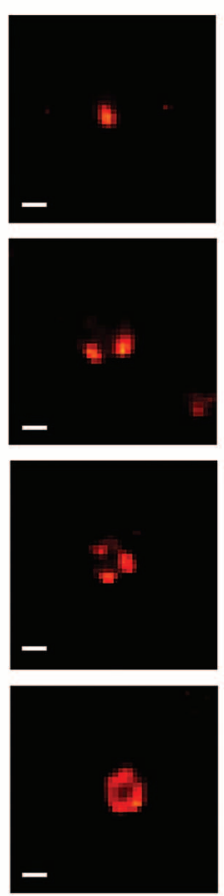

Env confocal signal
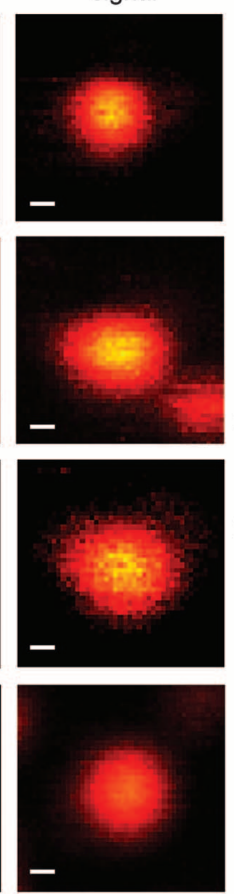

B

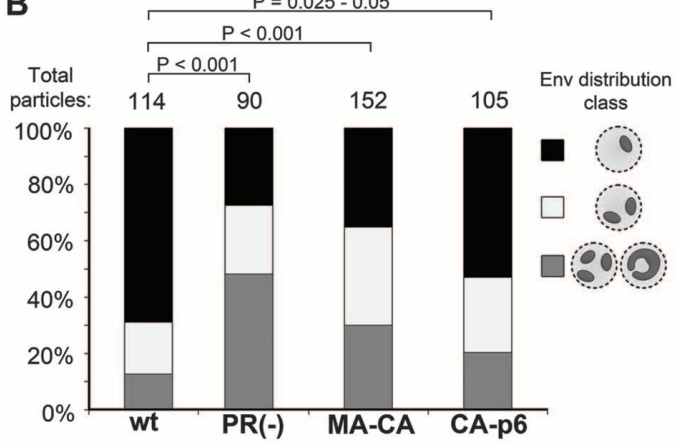

C

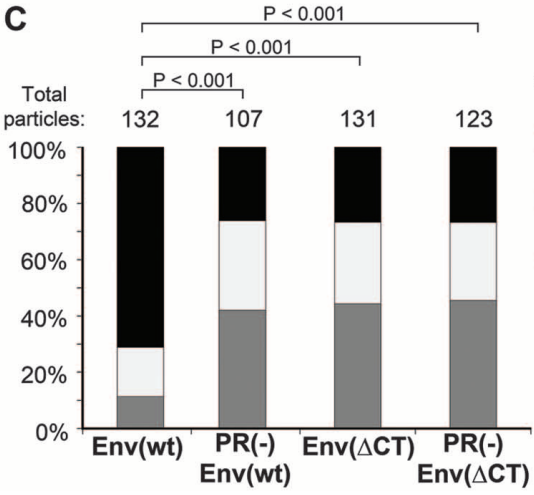

Env distribution class
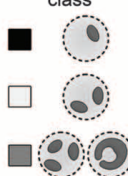

Fig. 4. Phenotype of HIV-1 variants and Env distribution of cell-attached virus particles. (A) Single-round virus infectivity. SupT1R5 cells were infected with equal amounts of HIV-1 carrying Env(wt) or $\operatorname{Env}(\Delta C T)$. HIV-1-positive cells were quantitated by flow cytometry. Mean \pm SD values from three independent experiments are shown with unpaired $t$ test for significance. (B) HIV-1 infectious titers determined by endpoint titration on C8166 cells. Mean \pm SD values from three independent experiments are shown with unpaired $t$ test for significance. (C) Cell entry efficiency. Equal amounts of mature or immature reporter particles carrying the indicated Env variants were used to infect primary lymphocytes. Intracellular reporter-mediated cleavage of the fluorescent substrate was measured by flow cytometry. Mean \pm SD values from three experiments using cells from three different donors are shown with unpaired $t$ test for significance. (D to G) Env distribution of cell-attached HIV-1. Particles containing eGFP.Vpr (green) were pre-incubated with 2 G12 Fab and allowed to attach to SupT1R5 cells followed by immunostaining for Env (orange) and cell surface CD4 (blue). Individual cell-attached particles were localized with the eGFP.Vpr signal in the confocal mode and Env/CD4 signals were analyzed by a dual-color
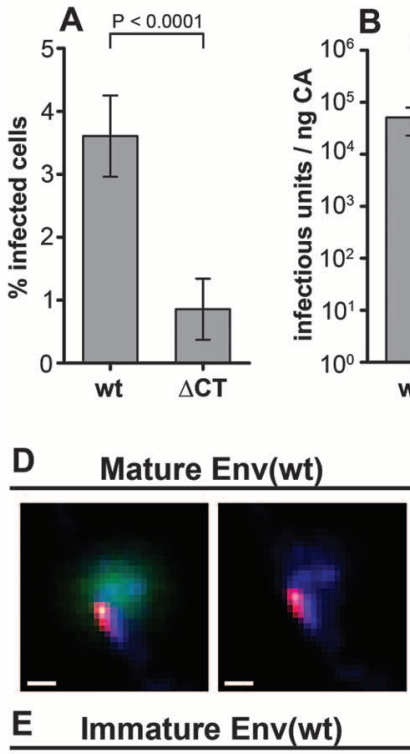
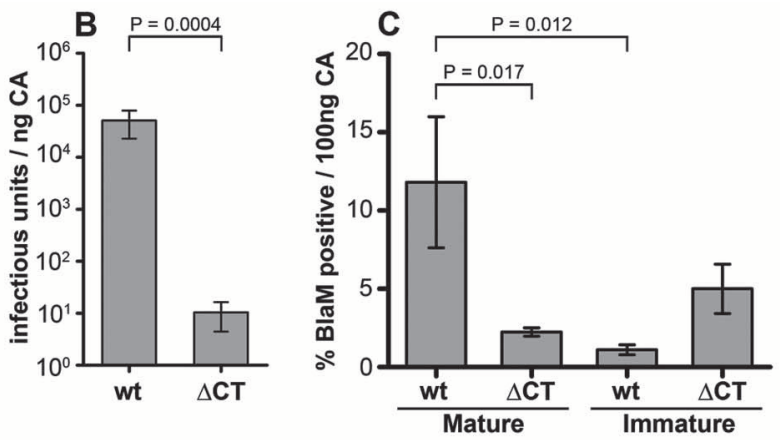

H
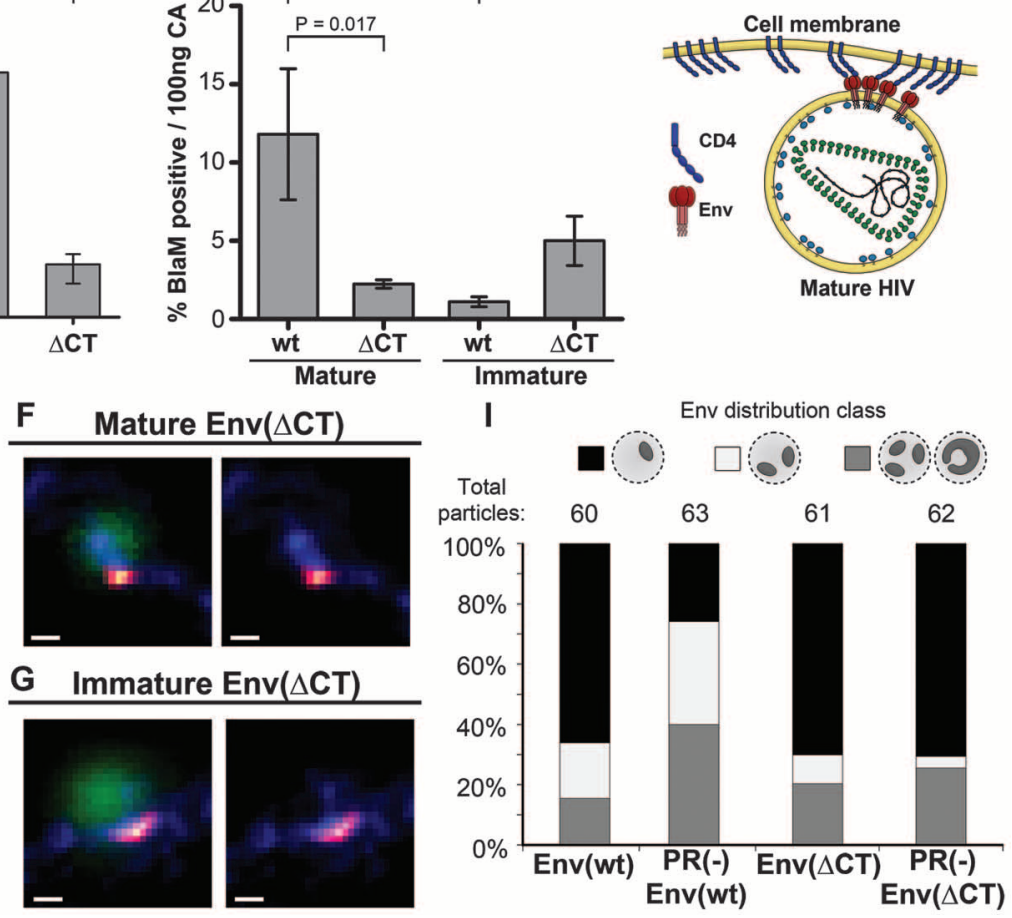

STED microscopy. Panels display representative images of the respective cellattached particles with eGFP/Env/CD4 signal overlay (left) or Env/CD4 signal overlay (right). Scale bars: $100 \mathrm{~nm}$. (H) Schematic illustration of a cell-attached mature HIV particle. (I) Env distribution patterns of cell-attached mature and immature (PR-) particles pseudotyped with $\operatorname{Env}(w t)$ or $\operatorname{Env}(\Delta C T)$ were classified as described in the legend to Fig. 3, B and C. 
The strength of the phenotype may thus be influenced by the relative incorporation of Env variants. This view is consistent with the finding that the $\operatorname{Env}(\Delta \mathrm{CT})$ variant shows a celltype-dependent infectivity defect $(3,22)$, which correlates with Env incorporation and can be rescued by a mutation that increases this incorporation (25).

We suggest that Env trimers are initially recruited to viral budding sites in a random distribution, yielding a multifocal appearance. Their lateral movement is restricted by the underlying rigid Gag lattice interacting with the Env $\mathrm{CT}$ and preventing formation of a single Env cluster in the immature virus. Proteolytic maturation, specifically the separation of MA from CA, overcomes this restriction, leading to coalescence into a single Env focus, driven by intermolecular Env CT interactions. This rearrangement polarizes the virus particle with subsequent attachment of the Env cluster to a CD4 patch on the target cell surface, thus initiating virus entry. Whereas Env trimers with truncated CT are mobile irrespective of Gag maturation, they lack the propensity to cluster. Clustering of Env trimers is partially rescued upon engagement of the cellular receptor provided that individual trimers are free to move within the viral membrane. Conversion of the inner core is thereby coupled to surface alterations in a mechanism of "inside-out signaling," ensuring that only particles whose interior has switched to the entry mode are fully competent for membrane fusion.

\section{References and Notes}

1. P. Cosson, EMBO J. 15, 5783 (1996).

2. E. O. Freed, M. A. Martin, J. Virol. 70, 341 (1996).

3. T. Murakami, E. O. Freed, Proc. Natl. Acad. Sci. U.S.A. 97, $343(2000)$.

4. E. Chertova et al., J. Virol. 76, 5315 (2002).

5. P. Zhu et al., Proc. Natl. Acad. Sci. U.S.A. 100, 15812 (2003).

6. R. H. Cheng et al., Cell 80, 621 (1995).

7. A. Harris et al., Proc. Natl. Acad. Sci. U.S.A. 103, 19123 (2006).

8. P. Zhu et al., Nature 441, 847 (2006).

9. A. Helenius, Cell 69, 577 (1992).

10. T. Murakami, S. Ablan, E. O. Freed, Y. Tanaka, J. Virol. 78, 1026 (2004).

11. D. J. Wyma et al., J. Virol. 78, 3429 (2004).

12. N. Kol et al., Biophys. J. 92, 1777 (2007).

13. S. W. Hell, ]. Wichmann, Opt. Lett. 19, 780 (1994).

14. A. Trkola et al., J. Virol. 70, 1100 (1996).

15. P. Roben et al., J. Virol. 68, 4821 (1994).

16. D. McDonald et al., J. Cell Biol. 159, 441 (2002).

17. J. A. Briggs, T. Wilk, R. Welker, H. G. Kräusslich, S. D. Fuller, EMBO J. 22, 1707 (2003).

18. T. Endress et al., Eur. Biophys. J. 37, 1291 (2008).

19. A. de Marco et al., PLoS Pathog. 6, e1001215 (2010).

20. M. J. Forster, B. Mulloy, M. V. Nermut, J. Mol. Biol. 298, $841(2000)$
21. S. F. Lee et al., J. Biol. Chem. 275, 15809 (2000).

22. T. Wilk, T. Pfeiffer, V. Bosch, Virology 189, 167 (1992).

23. M. Cavrois, C. De Noronha, W. C. Greene, Nat. Biotechnol. 20, 1151 (2002).

24. R. Sougrat et al., PLoS Pathog. 3, e63 (2007).

25. D. Holtkotte, T. Pfeiffer, T. Pisch, V. Bosch, AIDS Res. Hum. Retroviruses 22, 57 (2006).

26. L. A. Carlson et al., Cell Host Microbe 4, 592 (2008).

Acknowledgments: We are grateful to ]. Mak (Burnet Institute, Melbourne) for purified recombinant HIV-1 Gag and to P. Guardado-Calves (Institut Pasteur, Paris) for monomeric and trimeric Env gp140. We thank L. Castillo for purified recombinant HIV-1 CA, V. Bosch (DKFZ Heidelberg) for HIV-1 gp120 and CD4 antisera as well as HIV-1 Env expression plasmids, R. Doms (University of Pennsylvania) for SupT1R5 cells, C. Aiken (Vanderbilt University Medical Center) for infectious HIV-1 variant plasmids, and T. Hope (Northwestern University (hicago) for the eGFP.Vpr construct. H.-G.K. is an investigator of the Excellence Cluster CellNetworks funded by the German Research Foundation (DFG). S.W.H. holds a patent on STED microscopy in several European countries and the United States, which has been licensed to Leica, a microscope manufacturer.

\section{Supplementary Materials}

www.sciencemag.org/cgi/content/full/338/6106/524/DC1

Materials and Methods

Figs. S1 to S10

Table S1

References (27-33)

20 June 2012; accepted 6 September 2012

$10.1126 /$ science. 1226359

\section{In Vivo Architecture and Action of Bacterial Structural Maintenance of Chromosome Proteins}

\author{
Anjana Badrinarayanan, ${ }^{{ }^{*}} \dagger$ Rodrigo Reyes-Lamothe, ${ }^{{ }}{ }^{*} \ddagger$ Stephan Uphoff, ${ }^{2}$ \\ Mark C. Leake, ${ }^{2} \S$ David J. Sherratt ${ }^{1} \S$
}

SMC (structural maintenance of chromosome) proteins act ubiquitously in chromosome processing. In Escherichia coli, the SMC complex MukBEF plays roles in chromosome segregation and organization. We used single-molecule millisecond multicolor fluorescence microscopy of live bacteria to reveal that a dimer of dimeric fluorescent MukBEF molecules acts as the minimal functional unit. On average, 8 to 10 of these complexes accumulated as "spots" in one to three discrete chromosome-associated regions of the cell, where they formed higher-order structures. Functional MukBEF within spots exchanged with freely diffusing complexes at a rate of one complex about every 50 seconds in reactions requiring adenosine triphosphate (ATP) hydrolysis. Thus, by functioning in pairs, MukBEF complexes may undergo multiple cycles of ATP hydrolysis without being released from DNA, analogous to the behavior of well-characterized molecular motors.

$\mathrm{S}$ MC (structural maintenance of chromosome) complexes share conserved architectures and function in chromosome maintenance in all domains of life, although the molecular mechanism by which they act in vivo is unknown (1-3). In eukaryotes, SMC heterodimers associate with a range of accessory proteins, acting in chromosome organization, sister chromosome cohesion, and other chromosome biology functions, whereas in bacte- ria an SMC homodimer and associated accessory proteins act in chromosome maintenance (1). In Escherichia coli and some other $\gamma$ proteobacteria, a distant SMC relative, MukB with accessory proteins MukE and MukF, replaces the typical SMC complex but has similar functions $(4,5)$. Bacterial smc null mutants are frequently temperature sensitive, produce anucleate cells, and show disturbed chromosome organization at permissive temperature, indicating roles in SMC-mediated chromosome segregation and/or compaction $(1,6,7)$. In E. coli undergoing nonoverlapping replication cycles, MukBEF accumulates as "spots" at about one to three discrete chromosome locations, typically at mid-cell and/or quarter-cell, in the same regions as replication origins (6). Structural and biochemical MukBEF fragment studies report two subunit arrangements, $2: 4: 2$ or $2: 2: 1$, for MukB:E:F, dependent on whether adenosine triphosphate (ATP) is absent or bound, respectively (8) (fig. S1A). Here, our aim was to understand the molecular architecture of active SMC complexes in vivo, as well as the transformations undergone during ATP binding and hydrolysis, as complexes associate with, and dissociate from, the chromosome.

E. coli cells, in which endogenous MukBEF genes were replaced by functional yellow fluorescent protein (YPet) fusions, were analyzed by slimfield microscopy, a strategy used previously for studying replisomes (9) (Fig. 1A, figs. S1 and S2, and tables S1 to S3). Analysis of the numbers of MukB, E, or F molecules

${ }^{1}$ Department of Biochemistry, University of Oxford, UK ${ }^{2}$ Department of Physics, University of Oxford, UK.

*These authors contributed equally to this work. †Present address: Department of Biology, Massachusetts Institute of Technology, Cambridge, MA 02139, USA. $\ddagger$ Present address: Department of Biology, McGill University, Montreal, Quebec H3G 0B1, Canada.

§To whom correspondence should be addressed. E-mail: m.leake1@physics.ox.ac.uk (M.C.L.); david.sherratt@bioch. ox.ac.uk (D.J.S.) 